\title{
STARDUST MEMORIES: EL YO QUE RECUERDA, EXPERIENCIAS DE CONSUMO Y PATERNALISMO LIBERTARIO
}

\author{
Lluís Pla Vargas \\ SFPUB (Seminario de Filosofía Política de la Universitat de Barcelona) \\ lluisplavargas@gmail.com
}

\section{RESUMEN}

Este artículo revisa algunos de los hallazgos de la economía conductual con la finalidad de observar su aplicación a los ámbitos del consumo y la política. Por un lado, partiendo de la distinción entre un yo que experimenta las situaciones y un yo que las recuerda, se abre la posibilidad de explicar mejor el comportamiento cotidiano de los consumidores. Por otro lado, el refuerzo del yo que recuerda aparece como una estrategia básica de la política del paternalismo libertario, la cual pretende promover el bienestar de las personas tal como éstas lo querrían. No obstante, aunque se reconocen sus virtudes, este trabajo concluye presentando un argumento crítico contra un posible gobierno paternalista libertario.

Palabras clave: economía conductual, consumo, paternalismo, paternalismo libertario.

\section{STARDUST MEMORIES: THE REMEMBERING SELF, CONSUMPTION EXPERIENCES AND LIBERTARIAN PATERNALISM}

\section{Abstract}

This paper reviews some findings of Behavioural Economics in order to observe its application on the fields of consumption and politics. On one hand, starting from the difference between a self that experiences situations and a self that remembers them, it is an open possibility for explaining better consumer ordinary behaviour. On the other hand, the reinforcement of remembering self appears as a basic strategy of libertarian paternalism politics, which is intended to promote people's welfare as they would want to get it. However, even if admitting its virtues, this work concludes with a critical argument against a hypothetical libertarian paternalism government.

KEYWORDs: behavioural economics, consumption, paternalism, libertarian paternalism. 


\section{INTRODUCCIÓN}

En buena medida, los hallazgos de lo que se ha venido a denominar economía conductual -Behavioural Economics ${ }^{1}$ - han contribuido de manera decisiva a transformar la imagen de la acción y el pensamiento humanos construida sobre el modelo del agente económico racional. Estos descubrimientos han iluminado la existencia de "mapas de racionalidad limitada" y han fijado la existencia de sesgos sistemáticos que afectan a los razonamientos y las decisiones de los seres humanos con independencia de su grado de inteligencia. Una de sus tesis principales, defendida en particular por el psicólogo Daniel Kahneman, es que existe una discrepancia entre dos yo, el yo que experimenta y el yo que recuerda, los cuales no solamente operan de forma distinta, sino también contrapuesta. A pesar de que no resulta intuitivo, Kahneman ha defendido a partir de una base experimental que el responsable de las elecciones y la toma de decisiones es el yo que recuerda, pero ha añadido que éste obedece a un patrón que genera sesgos y elecciones erróneas. Según Kahneman, tres rasgos principales caracterizan este patrón: 1) su sensibilidad a la retrospección, 2) el olvido de la duración y 3) el énfasis en la regla del pico final.

Teniendo presente este esquema conceptual, este trabajo pretende exponer, en una primera sección, algunos de los descubrimientos de la economía conductual vinculados a la arquitectura de los denominados Sistema 1 y Sistema 2, respectivamente el sistema intuitivo y el reflexivo de los seres humanos, así como el patrón psicológico al que se atiene el yo que recuerda. A continuación, en una segunda sección, se revela la posibilidad de aplicar este patrón a una estructura de explicación de la acción de los consumidores mediante tres pasos: en primer lugar, se señala la conveniencia de desdoblar la experiencia del consumo entre aquélla que se lleva a cabo efectivamente y aquélla que se recuerda; en segundo lugar, se constata que los rasgos del patrón que sigue el yo que recuerda se aplican a las elecciones concretas de los consumidores; $y$, en tercer lugar, se propone la idea de que las decisiones del consumidor no se basan en la experiencia directa de los bienes y servicios que han de ser adquiridos, sino en el recuerdo, sometido a sesgos, de experiencias anteriores de consumo de bienes y servicios. Después, en una tercera sección, se presenta la conexión de todo este esquema conceptual con la propuesta del paternalismo liber$\operatorname{tario}^{2}$, que ha sido considerada como la teoría política más acorde con las enseñanzas de la economía conductual, con objeto de mostrar precisamente algunas de sus implicaciones ético-políticas. En una cuarta sección, se evalúa el concepto de paternalismo libertario en términos de un prometedor intento de corrección del comportamiento sesgado de los ciudadanos y/o consumidores erigido sobre el refuerzo del yo que recuerda. Por último, se abre a la discusión un argumento que persigue

1 Véanse, por ejemplo, dos excelentes compendios en Kahneman, D. Pensar rápido, pensar despacio, Penguin Random House, Barcelona, 2012 y Camerer, C., Loewenstein, G. y Rabin, M. (eds.) Advances in Behavioral Economics, Sage, Princeton, 2004.

2 Thaler, R. y Sunstein, C. Nudge. Un pequeño empujón, Taurus, Madrid, 2009; SunsTEIn, C. Paternalismo libertario. ¿Por qué un empujoncito?, Herder, Barcelona, 2017. 
examinar las limitaciones del paternalismo libertario como mecanismo de legitimación del poder.

\section{EL YO QUE RECUERDA}

En la película Stardust Memories, el cineasta Woody Allen interpreta a Sandy Bates, un famoso director de cine muy conocido por sus comedias que está sufriendo una profunda crisis existencial. Allen da vida a un personaje doblemente frustrado: por un lado, su vida personal se ciñe a relaciones superficiales e insatisfactorias y, por otro, se siente incomprendido como artista. No obstante, Bates acude a un lujoso hotel donde se le hace un cálido homenaje. En un momento de su caótica estancia, imagina o sueña que el homenaje prometido se le hace una vez que ha muerto. Al recibir un premio a la trayectoria de toda su vida artística, y verse en la obligación de dar un discurso frente a un auditorio entusiasta, Bates argumenta que, ante la perspectiva definitiva e irrevocable de la muerte, uno necesita desesperadamente agarrarse a algo. Y entonces rememora una sencilla escena doméstica. Es un domingo de primavera en Nueva York, siente cómo se aproxima el verano, suena Stardust de Louis Armstrong en el tocadiscos y, mientras se está comiendo un yogur, a unos pasos, su antigua novia, Dorrie, ojea el periódico tumbada en el suelo y le lanza una sonrisa. Bates -o Allen- dice entonces que la perfección de aquel instante le conmovió de una manera muy profunda, le hizo sentirse feliz, casi indestructible.

Podemos compartir perfectamente los rasgos de esta experiencia. Si echamos la vista atrás, todos y cada uno de nosotros también podemos identificar momentos de esta clase: aparentemente intrascendentes, cotidianos, pero cargados de un enorme significado para nuestras vidas. ¿Cuáles son las razones de esta clase de experiencias y de su profunda carga evaluativa? La psicología contemporánea ha demostrado que la relevancia que atribuimos a nuestros recuerdos no depende necesariamente de la experiencia realmente vivida, sino de la retrospección específica de esa experiencia que, en un momento concreto, lleva a cabo la memoria en el marco de nuestra trayectoria vital global. Pero al contenido de esta trayectoria vital global, obviamente, también accedemos mediante la memoria. De manera que, en cierto modo, la decantación de nuestros recuerdos otorga o sustrae sentido a nuestras vidas tal como nos las explicamos. Quizás la experiencia de Bates no fuese en realidad tan memorable -él no la evaluó así en aquel momento-, pero la memoria la situó posteriormente en un mosaico de recuerdos en el cual destacó por razones idiosincrásicas, esa clase de razones que solo el sujeto implicado que rememora podría finalmente comprender. En consecuencia, parece que el yo que recuerda las situaciones atiende a motivos distintos del yo que las experimenta.

Desde mediados de la década de 1970 hasta la actualidad, los hallazgos de la denominada economía conductual ${ }^{3}$ han contribuido a transformar la imagen de

3 Kahneman, D. y Tversky, A. «El juicio bajo incertidumbre: heurísticas y sesgos», 1974, Apéndice A, en Kahneman, D. Pensar rápido, pensar despacio, op. cit.; Kahneman, D. y Tversky, A. 
la acción humana erigida sobre el modelo estándar del agente económico racional. Como ha sido ampliamente reconocido, tales descubrimientos han revelado la existencia de una serie de "mapas de racionalidad limitada" ${ }^{4}$ han especificado una serie de sesgos sistemáticos que afectan a los razonamientos y las decisiones de los seres humanos al margen de su nivel de inteligencia. La versión más tradicional y grosera del modelo estándar del agente económico racional, a menudo reconocida bajo la expresión homo economicus, se aplicó no solo al estudio de la acción humana en el ámbito de la producción económica, sino también a su ejercicio en el del consumo. De una manera social y psicológicamente poco realista, esta versión supone que los agentes económicos no únicamente son resueltamente racionales y completamente egoístas, sino que además pueden captar y resolver los más difíciles problemas de optimización que se les puedan plantear ${ }^{5}$. Por consiguiente, el esquema, en su versión más burda, no contempla el impacto de las emociones sobre la cognición, la posibilidad de comportamientos altruistas ni tampoco la existencia de defectos o limitaciones en la comprensión de las situaciones por parte de los agentes económicos. Y, con todo, esta versión estándar se reveló como un esquema conceptual influyente, ya que fue usado de manera amplia y exhaustiva como trasfondo explicativo último en otras ciencias sociales, como la sociología y la ciencia política. De hecho, una de sus versiones refinadas ha llegado a alcanzar el estatus de una teoría independiente de la elección racional que, a pesar de contar con valedores potentes, como Kenneth Arrow, Frederick Schick o Jon Elster, fue asumiendo progresivamente algunas de las críticas recibidas y tornándose más sofisticada con el paso del tiempo ${ }^{6}$.

El primer trabajo importante conjunto de Kahneman y Tversky giró sobre las heurísticas y los sesgos que operan en los juicios realizados en condiciones de incertidumbre. Los autores mostraron que los individuos estiman la probabilidad de un evento incierto a partir de un número limitado de principios heurísticos -representatividad, disponibilidad y anclaje-, los cuales reducen la mucho más compleja y lenta tarea de estimación de probabilidades. Las heurísticas o atajos mentales suelen ser de utilidad para pensar rápido, pero el precio que se suele pagar por ello es la

«Elecciones, valores y marcos», 1983, Apéndice B, en Kahneman, D. Pensar rápido, pensar despacio, op. cit.; Camerer, C. y Loewenstein, G. «Behavioral economics: Past, present, future», en Camerer, C., Loewenstein, G. y Rabin, M. (eds.) Advances in Behavioral Economics, op. cit., pp. 3-51; Thaler, R. «Toward A Positive Theory of Consumer Choice», Journal of Economic Behavior and Organization, 1980, pp. 39-60; Thaler, R. Quasi-Rational Economics, Russell Sage Foundation, Nueva York, 1991; LevitT, S. y List, J. «Homo Economicus Evolves», en Science, 319, 2008, pp. 909-910.

${ }^{4}$ Kahneman, D. «Mapas de racionalidad limitada: psicología para una economía conductual», en Revista Asturiana de Economía, 28, 2003, pp. 181-225.

5 Cf. Levitt, S. y List, J. «Homo Economicus Evolves», op. cit., p. 909.

${ }^{6}$ El objetivo principal de este trabajo no es examinar la teoría de la elección racional-Rational Choice Theory- y sus limitaciones, sino las aportaciones potenciales de la economía conductual en los ámbitos del consumo y la política. Una revisión sucinta y útil de la teoría de la elección racional puede encontrarse en Aвitol, P. у Bотеro, F. «Teoría de elección racional: estructura conceptual y evolución reciente», Colombia Internacional, 62, 2005, pp. 132-145. 
recaída en apreciaciones erróneas sistemáticas ${ }^{7}$. En su segundo gran estudio inicial, la investigación giró sobre valores y marcos en las elecciones que se toman en contextos de riesgo. Sostuvieron entonces que los mismos problemas de toma de decisiones pueden ser descritos o enmarcados de múltiples maneras dando lugar a preferencias distintas, lo cual vulnera el criterio de invariancia de la elección racional. Por lo demás, demostraron la existencia de una "psicofísica del valor» según la cual los seres humanos sienten aversión al riesgo en un contexto de ganancias mientras buscan activamente el riesgo en un contexto de pérdidas ${ }^{8}$.

En esta temprana etapa de su programa de investigación, Kahneman y Tversky no sospechaban que, en realidad, lo que estaban examinando dentro de la teoría del juicio y de la decisión eran las consecuencias de una arquitectura cognitiva y emocional concreta. Más tarde, comprendieron que esta arquitectura cognitiva y emocional se constituye sobre la existencia de dos sistemas contrapuestos, pero estrechamente vinculados, a los cuales nos podríamos referir, sencillamente, como los sistemas de la intuición y el razonamiento complejo. Se trata, en suma, de dos funcionalidades de la misma mente a las cuales denominaron más tarde, siguiendo la terminología que habían empleado inicialmente los psicólogos Stanovich y West, Sistema 1 y Sistema 29 . Expondremos brevemente a continuación sus rasgos principales y su modo de funcionamiento.

El Sistema 1 opera de forma rápida y automática, con poco o ningún esfuerzo y sin que haya sensación de control voluntario por parte del agente. Se trata de la ventana intuitiva de la mente, a través de la cual circulan las impresiones y las sensaciones más inmediatas. El Sistema 1 está anclado en las operaciones de la memoria asociativa, la cual nos permite, en principio, conectar todo con todo ${ }^{10}$. El Sistema 1 nos permite llevar a cabo muchas y diversas acciones. Por ejemplo, nos permite conducir tranquilamente por un barrio conocido mientras mantenemos una conversación con nuestro acompañante en el coche, orientarnos hacia la fuente de un ruido, responder a la cuestión $" 2+2=\dot{\varepsilon}$ ?», poner cara de desagrado al ver una cucaracha, detectar hostilidad en una voz o una expresión, percibir que un objeto está más lejos que otro, completar expresiones como «Donde las dan...»o «Pan y...», etc.

El Sistema 2, en cambio, concentra la atención en determinadas actividades mentales como los cálculos complejos o el razonamiento verbal. El Sistema 2

7 Kahneman, D. y Tversky, A. «El juicio bajo incertidumbre: heurísticas y sesgos», 1974, Apéndice A en Kahneman, D. Pensar rápido, pensar despacio, op. cit.

8 Kahneman, D. y Tversky, A. «Elecciones, valores y marcos», 1983, Apéndice B, en Kahneman, D. Pensar rápido, pensar despacio, op. cit.

9 Cf. Stanovich, K. y West, R. «Individual Differences in Reasoning: Implications for the Rationality Debate», Behavioral and Brain Sciences, 23, 2000, pp. 664-665.

${ }^{10}$ No deja de ser curioso que la especificación de tal capacidad se aproxime a la descripción que ofreció el filósofo estadounidense Wilfrid Sellars de la actividad filosófica. El pasaje de Sellars reza: «The aim of philosophy, abstractly formulated, is to understand how things in the broadest sense of the term hang together in the broadest possible sense of the term». SelLars, W. «Philosophy and the Scientific Image of Man», Empiricism and the Philosophy of Man, Routledge and Kegan Paul, Londres, 1963, pp. 1-40. 
se activa en circunstancias en las cuales es imperativo concentrarse, actuar reflexivamente, $y$, a diferencia del Sistema 1, sigue reglas. El Sistema 2 también nos permite hacer muchas y diversas acciones, pero su sello característico es la lentitud, el progreso punto por punto, la minuciosidad. Por ejemplo, el Sistema 2 nos permite escuchar la voz de una persona conocida en un recinto atestado y ruidoso, observar un comportamiento adecuado en una reunión social, contar las veces que aparece la letra $a$ en la página de un texto, estacionar en una plaza de parking especialmente estrecha, comparar dos modelos de móvil y decidir finalmente cuál comprar, realizar un examen de oposición a notarías, comprobar la validez de un argumento lógico complejo, etc.

Cuando pensamos en nosotros mismos, solemos identificamos de manera aproblemática con el Sistema 2, con el yo supuestamente consciente, racional, que tiene creencias fundamentadas y toma decisiones en coherencia con ellas. Solemos, pues, identificarnos con la funcionalidad lenta de la mente, que, aparentemente, es la que se encarga de tomar las decisiones. Sin embargo, el Sistema 2 no está en el lugar donde se desarrolla la acción dramática de la vida. Allí se encuentra, en cambio, el Sistema 1, que, en conexión con la memoria asociativa, genera continuamente impresiones y sentimientos. Kahneman señala que tales impresiones y sentimientos «son las fuentes principales de las creencias explícitas y las elecciones deliberadas del Sistema $2{ }^{11}$. De modo que, normalmente, nos encontramos en primer lugar con un flujo de intenciones, sensaciones y emociones emitido por el Sistema 1 . Si el Sistema 2 les da su aprobación, entonces estas sensaciones y emociones se tornan en creencias y las intenciones en acciones voluntarias. Siempre que no haya acontecimientos que rompan este flujo y obliguen a pensarlo, o a repensarlo, el Sistema 2 acepta de buen grado todo lo que el Sistema 1 le presenta. Eso quiere decir que, generalmente, «damos crédito a nuestras impresiones y cumplimos nuestros deseos» ${ }^{12}$. Si surge un problema, entonces el Sistema 1 reclama la actuación del Sistema 2. Con otras palabras: «El Sistema 2 es activado cuando asistimos a un acontecimiento que altera el modelo del mundo que el Sistema 1 mantiene» ${ }^{13}$.

Otra de las tesis fundamentales de la economía conductual, defendida en particular por Kahneman, es la existencia de una profunda discrepancia entre dos yo, el yo que experimenta (experiencing self) y el yo que recuerda (remembering self), los cuales no solo operan de forma distinta, sino también contrapuesta. Para ilustrar la idea, imaginemos, en primer lugar, a un paciente expuesto a un tratamiento médico doloroso y, en segundo lugar, a ese mismo paciente evaluando su experiencia de ese tratamiento a posteriori. Podríamos ahora plantear si la intensidad del dolor experimentado por el paciente en el primer momento es idéntica a la intensidad del dolor recordado en el segundo momento. Alguien podría sugerir que las intensidades deben ser idénticas y que, si no se lo parece al paciente, no es porque sean dis-

\footnotetext{
${ }^{11}$ Kahneman, D. Pensar rápido, pensar despacio, op. cit., p. 35.

${ }^{12}$ Ibidem, p. 40.

13 Ibidem.
} 
tintas, sino porque éste no recuerda bien la intensidad del dolor experimentado. No obstante, éste no parece un planteamiento muy prometedor, ya que la pregunta que se hace el paciente cuando está sometido al tratamiento no es la misma que cuando lo está recordando. Como reflexiona Kahneman: «El yo que experimenta responde a esta pregunta: ‘¿Duele?’. El yo que recuerda responde a esta otra pregunta: ‘¿Cómo ha sido todo?' ${ }^{14}$. A partir de una base experimental, el experimento conocido como "de la mano fría», Kahneman defiende que el responsable de las elecciones es el yo que recuerda y añade que éste obedece a un patrón que suele generar sesgos y elecciones erróneas. Este patrón se caracteriza por tres rasgos principales: a) su sensibilidad a la retrospección, b) el olvido de la duración y c) el énfasis en la regla del pico final ${ }^{15}$. A continuación, vamos a examinar brevemente tales rasgos.

La memoria, con su capacidad retrospectiva, es una herramienta imprescindible para la adaptación al entorno y constituye uno de los factores fundamentales del aprendizaje no solo a nivel individual, sino también social. El conocimiento memorizado, especialmente si va unido a una impronta emocional, proporciona a los individuos un eficaz repertorio de depósitos de sentido. Tales depósitos también pueden ser identificados a nivel social bajo la forma de costumbres, normas o instituciones. Por consiguiente, puede afirmarse que la actividad de los seres humanos, tanto en su dimensión individual como social, se nutre del conocimiento acumulado en estos depósitos de sentido. En suma, la retrospección de experiencias anteriores llevada a cabo por la memoria ofrece el conjunto general de hechos y criterios mediante cuyo examen se toman decisiones con objeto de minimizar errores de apreciación y conductas poco adaptativas.

El tiempo es uno de los factores fundamentales que se suele tener en cuenta a la hora de evaluar una experiencia dolorosa o placentera. Se suele dar por sentado que es preferible una experiencia placentera larga a una corta, así como resulta preferible una experiencia dolorosa corta a una larga. No obstante, el registro de experiencias dolorosas diversas recogido por médicos y psicólogos - por ejemplo, la de una colonoscopia sin anestesia- señala que los pacientes acusan de olvido de la duración, esto es, la duración del procedimiento doloroso no es un factor decisivo en las estimaciones posteriores del dolor total. Así pues, en consecuencia, las estimaciones de la experiencia dolorosa por parte de los pacientes no tienen tanto en cuenta el tiempo total -que es lo que, probablemente, tendría presente un observador externo en actitud objetivante-, sino otros factores subjetivamente relevantes; en particular, cómo se ha desarrollado generalmente ésta y cómo se ha vivido su momento final.

Son estas dos estimaciones las que constituyen la denominada regla del pico final. Ya sea que estemos hablando de situaciones dolorosas o placenteras, la cláusula «el modo en que se ha desarrollado la experiencia» suele tener como referencia ambas apreciaciones subjetivas: de un lado, el valor medio del nivel de dolor o de placer experimentado respectivamente en el peor o el mejor momento del proceso

\footnotetext{
${ }^{14}$ Ibidem, p. 496.

${ }^{15}$ Cf. ibidem, pp. 491-507.
} 
y, del otro, el valor medio del nivel de dolor o placer experimentado en el último momento del proceso. Por ejemplo, si el proceso doloroso tiene un nivel medio alto y un final abrupto y doloroso, se lo recordará como mucho peor a pesar de haber sido corto; en cambio, si el proceso doloroso tiene un nivel medio moderado y un final progresivamente menos doloroso, entonces se lo recordará mejor a pesar de haber sido largo.

Ahora bien, el yo que recuerda, que es en definitiva el yo que realiza las estimaciones, está sometido a sesgos. Si recordamos una experiencia que concluyó con un final desagradable, y si padecemos el olvido de su duración, entonces es muy probable que su estimación posterior la considere como globalmente negativa a pesar de que, tal vez, la experiencia fue en cambio positiva durante la mayor parte del tiempo. La experiencia de una tensa separación matrimonial puede arruinar toda posibilidad de salvar para el recuerdo los momentos en que la pareja fue feliz ${ }^{16}$. Algo parecido puede suceder con una semana de vacaciones que se arruina los dos últimos días debido al mal tiempo. En tales ejemplos se produce, por tanto, una divergencia clara entre el testimonio del yo que experimenta la situación presente y la evaluación de ésta por parte del yo que recuerda. Así pues, en conclusión, el yo experiencial está absorbido por la experiencia y, como sugiere Kahneman, carece de voz; pero, por otra parte, el yo mnémico puede equivocarse en sus evaluaciones a posteriori, si bien «es el único que registra y ordena lo que aprendemos de la vida, y el único que también toma decisiones» ${ }^{17}$.

\section{EXPERIENCIAS DE CONSUMO}

Ya se ha hecho referencia a la idea de que los trabajos de la economía conductual, con su visión psicológicamente más realista de los seres humanos, han tenido aplicación fuera del ámbito de su disciplina académica originaria. Pero más allá de la psicología, la economía, la sociología, la ciencia política, la estrategia militar y la filosofía, sus descubrimientos se han trasladado también al estudio del consumo con objeto de lograr que los consumidores tomen mejores decisiones, las políticas gubernamentales de protección al consumidor sean más eficaces $\mathrm{y}$, también, algunas de las peores consecuencias de deterioro medioambiental acarreadas por el consumo desmedido e irracional puedan ser evitadas o minimizadas ${ }^{18}$. En gene-

${ }^{16}$ Un conocido chiste reconoce este hecho. La esposa le dice al esposo: «¿Recuerdas lo felices que éramos hace 25 ańos?» Y el esposo, rascándose la cabeza, responde: «¿Hace 25 años? Pero si entonces no nos conocíamos». A lo que la mujer replica: «Por eso éramos felices».

${ }^{17}$ Kahneman, D. Pensar rápido, pensar despacio, op. cit., p. 496.

18 Por mencionar solamente algunos trabajos de la literatura de economía conductual sobre los aspectos mencionados, cuyo volumen, por lo demás, está creciendo de manera exponencial, véanse FoxalL, G. «Foundations of consumer behaviour analysis», Marketing Theory, vol. 1(2), 2001, pp. 165-199; DiClemente, D. y Hantula, D. «Applied behavioral economics and consumer choice», Journal of Economic Psychology, 24, 2003, pp. 589-602; Camerer, C. y Loewenstein, G. «Behavioral 
ral, estos ambiciosos objetivos se han perseguido empleando, fundamentalmente, a) la distinción de los Sistemas 1 y 2, b) un análisis de cómo se generan en la actividad del consumo los sesgos y principios heurísticos que fueron abordados por Kahneman, Tversky y Thaler en las primeras etapas de sus investigaciones -esto es, el sesgo del statu quo, el efecto dotación, la falacia del coste irrecuperable, la heurística de la disponibilidad, los efectos de enmarcado, la heurística del anclaje, etc.- y c) un examen del particular marco de elección en el cual se desarrolla la actividad de búsqueda, compra y uso de bienes y servicios.

No obstante, en esta profusa literatura, la distinción de Kahneman entre el yo que experimenta (experiencing self) y el yo que recuerda (remembering self) no ha encontrado acomodo explícito como estructura explicativa de la conducta del consumidor, sino solamente como presupuesto a tener en cuenta con vistas a la implementación de las políticas del denominado paternalismo libertario ${ }^{19}$. A continuación, procederemos a argumentar que es posible implementar esta distinción como una estructura básica para el estudio de las experiencias cotidianas de consumo de bienes y servicios. Tal implementación asume ciertos presupuestos. En primer lugar, la propuesta admite considerar la experiencia subjetiva del consumidor como un objeto central del análisis de modo análogo a como se tenía en cuenta la experiencia y su evaluación subjetivas en el caso de los individuos sometidos al experimento de la mano fría. En segundo lugar, la propuesta entraña que tal experiencia no puede reducirse a la mera actividad adquisitiva de compra y disfrute de bienes o servicios, que ha sido la caracterización frecuente que ha recibido desde el punto de vista de la economía tradicional, sino que, por el contrario, el consumo de bienes y servicios debe ser comprendido como «una actividad social cuantitativamente y cualitativamente central en nuestro actual contexto histórico $»^{20}$, una actividad, en definitiva, que desarrolla un papel central en el sistema informativo en expansión de esta sociedad en la cual bienes y servicios resultan «necesarios para hacer visibles y estables las categorías de una cultura ${ }^{21}$. Y, en tercer lugar, la propuesta reconoce que la experiencia social del consumo es una experiencia activa, no pasiva, en la cual se incluyen necesariamente elementos derivados de la clase social y de su gusto cultural prototípico, una experiencia que se constituye como una tarea de apropiación o, más bien, como sostiene Bourdieu, una tarea «en que el consumidor contribuye a producir el producto que consume al precio de un trabajo de localización y desciframiento ${ }^{22}$.

economics: Past, present, future», Camerer, C., Loewenstein, G. y Rabin, M. (eds.) Advances in Behavioral Economics, op. cit., pp. 3-51; MARTEAU, T. «Towards environmentally, sustainable human behaviour: targeting non-conscious and conscious processes for effective and acceptable policies", Philosophical Transactions of Royal Society, 2017, A 375; ReIsch, L. y ZhaO, M. «Behavioural Economics, Consumer Behaviour and Consumer Policy: State of The Art», Behavioural Public Policy, vol. 1, 2, 2017, 190-206; Sunstein, C. Paternalismo libertario. ¿Por qué un empujoncito?, op. cit.

${ }^{19}$ En particular, Sunstein, C. Paternalismo libertario. ¿Por qué un empujoncito?, op. cit.

20 Alonso, L.E. La era del consumo, Alianza, Madrid, 2005, p. 30.

${ }^{21}$ Douglas, M. e Isherwood, B. El mundo de los bienes, Grijalbo, México D.F., 1990, p. 74.

22 Bourdieu, P. La distinción. Criterios y bases sociales del gusto, Taurus, Madrid, 2006, p. 98. 
La referencia al carácter cotidiano de las actividades de consumo es relevante. La gran mayoría de las experiencias de consumo son actividades de gasto bajo o moderado que se repiten en contextos muy similares. La compra semanal en el supermercado del barrio sería el ejemplo paradigmático. En tales casos, si no se presentan factores como un bajo nivel de ingresos o una aguda conciencia ideológica que empuja a adquirir lo que se considera estrictamente necesario -lo que obliga a una preparación, revisión y ajuste rigurosos de la lista de la compra-, el nivel de atención medio de los consumidores es bajo, a la altura del carácter rutinario de la experiencia, la cual, normalmente, se desea rematar cuanto antes. Pero también se producen experiencias de consumo extraordinarias, que involucran gastos muy elevados y marcos de elección novedosos, aunque éstas suelen vivirse pocas veces en la vida. La compra de una casa, quizás la de un coche, o la contratación de un largo viaje transoceánico podrían ser ejemplos de esta índole. En tales casos, el nivel de atención medio de los consumidores suele ser más alto, éstos suelen estudiar las opciones con más detenimiento y, en general, toman sus decisiones después de una deliberación a menudo compartida con las personas que consideran importantes en sus vidas; todo ello, sin embargo, no elimina la existencia de errores de apreciación y decisiones equivocadas. En un perspicaz enfoque antropológico sobre el consumo, Mary Douglas y Baron Isherwood establecieron cómo esta distinción entre actividades de alta y baja frecuencia marca con claridad una distinción social entre actividades de alto y bajo estatus y, al tiempo, una distinción cultural en relación con el valor de los objetos involucrados en ellas ${ }^{23}$. La propuesta que aquí avanzamos tiene como ámbito de aplicación único las actividades de consumo de alta frecuencia y bajo estatus que implican bienes y servicios corrientes. Quedaría por investigar la posibilidad de extenderla a las actividades de consumo de baja frecuencia y alto estatus, como, por ejemplo, la compra de una casa, el pago por la organización de un banquete de boda o la adquisición de joyas.

La distinción de Kahneman entre el yo experiencial (experiencing self) y el yo que recuerda (remembering self) permite, en primer lugar, desdoblar la experiencia del consumo en dos momentos distintos: en el momento 1, la experiencia incorpora la incitación, la búsqueda, la adquisición y el uso del bien o servicio; en el momento 2, el consumidor recuerda el momento 1 en todas sus fases y hace una estimación de su valor como una experiencia globalmente positiva o negativa. Si la estimación es globalmente positiva, entonces el consumidor podría desear reproducirla no únicamente teniendo presente el bien adquirido o el servicio empleado, sino también el contexto espacial o temporal en que se realizó la adquisición o los caminos a través de los cuales se descubrió que se trataba de un bien o un servicio apreciables. Si la estimación es globalmente negativa, entonces es altamente probable que el consumidor no desee reproducir la experiencia e incluso se muestre precavido en los contextos espacial y temporal en los que tuvo lugar.

${ }^{23}$ Douglas, M. e Isherwood, B. El mundo de los bienes, op. cit. 
No obstante, tales estimaciones, como se ha indicado, acusan la acción de los sesgos. Por ejemplo, todo el mundo entiende que haya gente que frecuente a menudo los mismos restaurantes, pese a su comida y servicio regulares, y también que haya gente que no regrese nunca al restaurante donde, cierto día, les ofrecieron un servicio y una comida regulares. En todo caso, parece claro que lo que decide la reproducción de la experiencia de consumo con respecto a los mismos bienes o servicios y a los mismos contextos en que tuvo lugar es la estimación del yo que recuerda, no la experiencia directa del yo cuando está consumiendo. De hecho, la importancia del recuerdo, de un buen recuerdo, está también presente en los esfuerzos más o menos conscientes que llevan a cabo los propietarios de los negocios y los expertos en marketing para lograr que el consumidor se marche a casa con un buen sabor de boca. Un buen recuerdo es, en cierto modo, un regalo -de ahí la analogía entre ambos términos-; se trata, en primera instancia, de un regalo para el consumidor, pero es también, en segunda instancia, un regalo para el vendedor, que está interesado en que el primero regrese.

Podría objetarse que esta distinción entre los dos momentos de la experiencia del consumo se difumina en el caso del consumo de servicios, pues, a diferencia de lo que sucede con el de los bienes, adquisición y uso se producen simultáneamente. Así pues, la objeción plantearía que no hay razón para distinguir entre un momento de experiencia y otro de evaluación en el caso de los servicios porque podemos estimar si el servicio es bueno o malo durante los mismos momentos en que lo estamos recibiendo. Como en el caso de los bienes, evaluar un servicio implica usarlo, pero la única diferencia con respecto a la estimación del consumo de bienes consiste en la reducción del lapso temporal entre el momento del uso y el momento de su evaluación, no en su eliminación. Por lo demás, y en cualquier caso, el yo que recuerda seguirá teniendo la última palabra cuando el consumidor se plantee reproducir el consumo de un servicio concreto, ya sea tomar un taxi en lugar del autobús, cenar en un restaurante un poco más caro en lugar de hacerlo en uno más económico o escoger un paquete cerrado de viaje de vacaciones en el cual las visitas y las comidas están programadas en lugar de uno más abierto y flexible que permite una mayor libertad a los viajeros.

Establecida esta distinción entre el yo que consume y el yo que recuerda su consumo -es decir, la distinción entre un yo que, al consumir, no puede explicar su experiencia de consumo, y un yo que, sin consumir, orienta las decisiones de consumo posteriores-, y habiendo dejado claro que el yo que recuerda es el que determina, mediante su evaluación retrospectiva, el carácter de la experiencia de consumo y, por tanto, la probabilidad de su repetición, deben ahora considerarse los rasgos característicos de esa evaluación retrospectiva: por un lado, el olvido de la duración $y$, por el otro, la regla del pico final. Antes de entrar en ello, empero, cabría hacer una advertencia. Las evaluaciones retrospectivas no pueden ser reducidas a meras estimaciones llevadas a cabo en actitud objetivante y distanciada. Tal sería, de hecho, el formato de las estimaciones que podrían deducirse del modelo del homo economicus, pero no de la versión modificada, psicológica y sociológicamente densa, que se puede deducir de las investigaciones de la economía conductual. Para comprender el carácter real de las estimaciones que llevan a cabo las personas de sus expe- 
riencias pasadas, hay que incluir necesariamente elementos personales y contextuales: el influjo del temperamento, el grado de sensibilidad y la influencia del contexto desde el cual se realiza la propia evaluación retrospectiva.

Experiencias placenteras o dolorosas de la misma duración e intensidad pueden ser evaluadas de modo distinto por personas diferentes en función de su temperamento y su grado de sensibilidad. Una espera prolongada en un restaurante puede resultar más o menos tolerable no solo en función del hambre acumulada, sino también del temperamento, el estado de ánimo y de si la espera tiene lugar en una época de trabajo o de vacaciones. Por otra parte, el contexto social y personal desde el cual se realiza la estimación también resulta muy relevante, puesto que puede decantar la valoración en un sentido u otro. Por ejemplo, una persona que ha ascendido socialmente y lleva una vida desahogada puede que recuerde como una aventura simpática la época en que le resultaba complicado llegar a fin de mes y, en cambio, si no hubiera disfrutado de tal ascenso social, cabría considerar probable una valoración no tan positiva. $\mathrm{O}$, por otra parte, una persona jubilada podría valorar como placentera y positiva una época anterior de su vida en que llevaba a cabo muchas actividades a lo largo del día sin que a su valoración se sume el recuerdo de la gran carga de trabajo que tenía entonces y que, probablemente, le agobiaba ${ }^{24}$.

Así pues, la estimación del valor de una experiencia de consumo suele tener un carácter global e impresionista y en ella entran en juego los elementos de la personalidad mencionados - temperamento, sensibilidad-y el contexto social y personal desde el cual se hace la estimación. Pero es la particular combinación de estos elementos con los dos rasgos objetivables que se deducen del experimento de la mano fría, esto es, el olvido de la duración y la regla del pico final, lo que genera en cada caso la evaluación de la experiencia de consumo. Tomemos el ejemplo cotidiano y paradigmático: la visita al supermercado para hacer la compra semanal. Si el recuerdo de esa visita obtiene una valoración global positiva por parte del consumidor en todo aquello que le resulta subjetivamente relevante -proximidad, precios, comodidad, atención-y si el recuerdo queda fijado por un buen final de la visita - un descuento, un obsequio, un abono de 2 por 1, la sonrisa de la cajera o el mero aroma de la sección de panadería situada a la entrada del supermercado-, entonces es probable que el consumidor no tenga en cuenta en su recuerdo el largo rato que se ha demorado haciendo la compra y que, en caso de necesitarlo, vuelva a hacer la compra semanal en ese mismo lugar. Podemos tomar otro caso relacionado con el consumo de ocio. Aunque a algunas personas les pueda resultar incomprensible, no es extraño que haya mucha gente dispuesta a hacer tres cuartos de hora de cola para poder disfrutar de un trayecto en una montaña rusa que dura aproximadamente dos minutos; el hecho de que deseen repetirla indica que el valor medio que otorgan a la experiencia y el valor del nivel de su pico final les resultaron suficientemente satisfactorios como para olvidar los 45 minutos de cola previos.

${ }^{24}$ Este aspecto ya fue captado por Cicerón: «Dulce es el recuerdo de los trabajos pretéritos». Cicerón, T. Etica para cada día, Península, Barcelona, 2000, p. 56. 


\section{UNA POLÍTICA PARA LA ECONOMÍA CONDUCTUAL}

El paternalismo libertario puede ser descrito como la teoría política que pretende responder a los progresos de la economía conductual promoviendo el bienestar de agentes a los cuales considera no enteramente soberanos sobre su cuerpo y mente, pero respetando sus fines en último término ${ }^{25}$. A pesar de que esta descripción pueda connotar que la teoría tiene un carácter novedoso, lo cierto es que ésta se ha articulado a partir de un viejo interés en el marco de la tradición liberal por el concepto de paternalismo ${ }^{26}$. En términos generales, las discusiones contemporáneas sobre el paternalismo suelen partir del conocido pasaje de John Stuart Mill, expuesto en Sobre la libertad, según el cual el poder público, sea el de la sociedad, sea el del gobierno, actúa legítimamente cuando coarta la libertad del individuo para evitar que dañe al prójimo, pero actúa ilegítimamente si interfiere en la libertad del individuo cuando el único afectado por las acciones de éste sea él mismo. Mill entendió que éste es el único y sencillo principio que debería guiar siempre la acción de los poderes públicos. El texto en cuestión es el siguiente:

... la única finalidad por la cual el poder puede, con pleno derecho, ser ejercido sobre un miembro de una comunidad civilizada contra su voluntad, es evitar que perjudique a los demás. Su propio bien, físico o moral, no es justificación suficiente [...]. La única parte de la conducta de cada uno por la que él es responsable ante la sociedad es la que se refiere a los demás. En la parte que le concierne meramente a él, su independencia es, de derecho, absoluta. Sobre sí mismo, sobre su propio cuerpo y espíritu, el individuo es soberano ${ }^{27}$.

Cuando Gerald Dworkin examina este pasaje, señala que, a diferencia de lo sostenido por Mill, en realidad, aquí no nos encontramos con un principio, sino con dos: un principio que afirma que la evitación del daño al prójimo es una justificación suficiente para la actuación legítima del poder contra la voluntad del individuo, y otro principio, de carácter distinto, que sostiene que el propio bien del individuo -su preservación, su promoción- nunca es una justificación suficiente para la actuación coercitiva del poder contra su voluntad ${ }^{28}$. Desdoblados de este modo, podría decirse que el primer principio justifica la actuación del poder público sobre el individuo a partir de consideraciones utilitaristas, ya que procura reducir el daño potencial que el individuo podría causar a los demás, mientras que el segundo principio prohíbe la actuación del gobierno a partir de un criterio distinto, no utilitarista, que estipula que la elección libre del individuo en asuntos que le atañen sola-

25 Thaler, R. y Sunstein, C. Nudge. Un pequeño empujón, op. cit.; Sunstein, C. Paternalismo libertario. ¿Por qué un empujoncito?, op. cit.

${ }^{26}$ Mill, J.S. Sobre la libertad, Alianza, Madrid, 2001; Dworkin, G. «Paternalism», en Sartorius, R. Paternalism, University of Minnessota Press, Minneapolis, 1983, pp. 19-34; Rawls, J. Una teoria de la justicia, FCE, México, D.F., 1995, pp. 234-235.

${ }_{27}$ Mill, J.S. Sobre la libertad, op. cit., p. 68.

${ }^{28}$ Dworkin, G. «Paternalism», en Sartorius, R. Paternalism, op. cit., p. 19. 
mente a él tiene un valor absoluto, un valor que es independiente de las consecuencias que ello le pueda generar. Es claro que la objeción más potente de Mill contra el paternalismo se apoya precisamente en este segundo principio, que descansa, como arguye Dworkin, «no en los bienes a los cuales conduce la libre elección, sino en el valor absoluto de la elección misma ${ }^{29}$. Desde el punto de vista de Mill, el paternalismo resulta inaceptable porque se propone interferir en la libertad y las decisiones de individuos maduros y soberanos, individuos capaces y legitimados para actuar como les plazca y en el sentido que les plazca en relación con sus cuerpos y sus mentes. Para Mill, en definitiva, el paternalismo es inadmisible porque vulnera el carácter autónomo y soberano del ser humano.

Tradicionalmente, el paternalismo ha sido entendido como la actitud que propone o sugiere un conjunto de medidas dirigidas a preservar o promover el bienestar de las personas tal como éstas lo querrían en aquellos casos en que están incapacitadas temporal o permanentemente para hacerlo por sí mismas. John Rawls, por ejemplo, se refiere al paternalismo como la necesidad de «escoger por otros teniendo una razón para creer que así escogerían ellos mismos si tuviesen uso de razón y pudiesen decidir de manera racional $\aleph^{30}$. Aunque la actuación paternalista reposa sobre la presuposición de una carencia de racionalidad temporal o permanente en aquéllos sobre los cuales se va a ejercer, lo cierto es que puede haber casos en los cuales resulta plausible que alguien decida racionalmente ser tratado de manera paternalista. Existen ciertas situaciones en las cuales puede ser racional que «un individuo acuerde con otros que le fuercen a actuar de una manera que, en el momento de la acción, puede no ver como deseable ${ }^{31}$. Un ejemplo clásico lo proporciona la Odisea de Homero. Se trata de un pasaje en el cual el protagonista de la historia, Odiseo, hace a sus marineros la petición de que, ante la perspectiva de encontrarse con las sirenas, cuyos cantos enloquecen a los hombres, lo aten al mástil de la nave y que, cuando los cantos de las sirenas los alcancen, no lo desaten por mucho que lo exija y los amenace con castigos severos en caso de no obedecerle. Podemos llamar a este tipo de medidas estrategias de precompromiso. Un documento de voluntades anticipadas es también una estrategia de precompromiso; en este caso, una persona capaz de tomar una decisión de manera libre y con la información suficiente comunica qué criterios y medidas se le deberían aplicar en caso de que por motivos físicos o psicológicos no pudiera expresar su voluntad en el momento de recibir un tratamiento médico concreto.

En el marco de la tradición liberal, la idea de que el gobierno pueda imponer medidas paternalistas a los ciudadanos ha sido justificada de dos maneras diferentes: una que avala la imposición de costes materiales sobre las elecciones de las personas con objeto de promover su bienestar - paternalismo duro- y otra que pretende incrementar el bienestar de las personas a través de una serie de estrategias que

\footnotetext{
29 Ibidem, p. 28.

30 Rawls, J. Una teoría de la justicia, op. cit., p. 199.

31 Dworkin, G. «Paternalism», en Sartorius, R. Paternalism, op. cit., p. 29.
} 
no implique cargar sus elecciones con costes materiales - paternalismo suave $e^{32}-$. Por consiguiente, el paternalismo suave busca influir en las creencias y la conducta de la gente con objeto de evitar que su bienestar a largo plazo se vea seriamente perjudicado y, para ello, emplea medidas de divulgación y recordatorio de informaciones relevantes, así como reglas por defecto. Ejemplos de recordatorios son las advertencias acerca del riesgo de muerte debido al consumo de tabaco incorporadas en los paquetes de cigarrillos o las pirámides de alimentación saludable que se suelen ver en las paredes de hospitales, centros cívicos y otros espacios públicos. Un ejemplo de regla por defecto sería la legislación ya impulsada en países como Francia, Chile, País de Gales u Holanda sobre la donación de órganos según la cual todo el mundo es donante de órganos si en vida no ha hecho constar lo contrario.

En el ámbito particular del derecho del consumo, se sostiene que si un artículo ostenta un precio concreto, entonces es una regla por defecto que el consumidor no deba hacer pagos adicionales al margen del precio ya indicado. En este mismo ámbito, la legislación contempla un periodo de garantía de dos años a contar desde el momento de la entrega del producto. En este plazo, pero principalmente en los seis primeros meses del mismo, existe una presunción a favor del consumidor y éste tiene derecho a la reparación, sustitución, rebaja en el precio o devolución si el producto presenta defectos o falta de adecuación. Como regla por defecto, se considera que cualquier contrato de compraventa que vulnere estas cláusulas se considera nulo, a pesar de que pueda haber sido establecido mediante consentimiento libre y expreso de las partes, ya fuese porque el consumidor pretendía obtener el producto más barato, ya porque el vendedor necesitaba deshacerse rápidamente de un stock.

El paternalismo suave usa habitualmente las advertencias, los recordatorios y las reglas por defecto y, en ese sentido, incluye lo que Cass Sunstein y Richard Thaler han denominado «empujoncitos» (nudges). Los «empujoncitos» no pretenden vulnerar en ningún caso las preferencias últimas de la gente, sino que intentan únicamente recordarles que puede haber mejores modos de promoverlas a largo plazo. Por este motivo, el paternalismo suave puede ser comprendido como un paternalismo libertario. Así pues, como ya se ha sugerido, la justificación del paternalismo libertario se apoya en su pretensión de promover el bienestar de las personas tal como ellas lo querrían a largo plazo, sin imponer costes materiales a sus elecciones y respetando, por tanto, su libertad de decisión en última instancia.

La reflexión de Sunstein a favor del paternalismo libertario también comienza con una discusión del pasaje de Mill. De entrada, Sunstein indica que la potente presuposición que hace Mill, esto es, que los individuos son quienes mejor conocen sus propios fines y que, por lo tanto, son los mejores jueces para saber qué medios hace falta implementar para alcanzarlos, no puede ser abordada como una cuestión filosófica, sino empírica. En general, puede afirmarse que la persona suele conocerse mejor a sí misma de lo que lo podrían hacer los demás, pero, aun así, no siempre elige bien. Si hay personas que emprenden cursos de acción o eligen cosas que menosca-

${ }^{32}$ Cf. Sunstein, C. Paternalismo libertario. ¿Por qué un empujoncito?, op. cit., p. 82. 
ban su bienestar a largo plazo, acciones o cosas que en ningún caso a la larga querrían, entonces es claro que la presuposición que subyace al principio de Mill no puede mantenerse en todos los casos. De hecho, con mucha frecuencia observamos que las personas son impulsivas, priorizan el corto plazo, no atienden a un rasgo relevante de la situación o del producto que le ofrecen, no leen la letra pequeńa, no hacen el cálculo demorado de las opciones que tienen y privilegian en cambio una heurística de la afectividad, o tienen expectativas excesivamente optimistas acerca de las situaciones en las que se encuentran o los objetos que adquieren. Constatados estos hechos, Sunstein se ve obligado a rechazar el argumento epistémico presupuesto por Mill, no porque en todos los casos sea erróneo, sino porque no siempre es correcto, y a validar una apuesta regulatoria razonable que pretenda o bien evitar las peores consecuencias de las decisiones erróneas o bien minimizar su impacto en el bienestar a largo plazo de la gente. De ahí que sostenga: «Mi afirmación central es simple: los fallos conductuales del mercado son un complemento importante a la explicación de los fallos del mercado y, en principio, justifican las respuestas (ideales) incluso si estas respuestas son paternalistas" ${ }^{33}$.

Pero, más allá de los fallos conductuales de los sujetos, el paternalismo libertario focaliza también la atención sobre algo más, algo quizás decisivo: la arquitectura de la elección. Esta arquitectura es un factor clave para analizar cómo se toman decisiones y para evaluar si éstas serán provechosas o perjudiciales a largo plazo para el bienestar de las personas tal como ellas mismas lo querrían. Por arquitectura de elección cabe entender el inevitable contexto material, social y cultural en el cual los individuos se ven obligados a tomar sus decisiones, un contexto que puede ser simple y beneficioso o bien complejo y gravoso. En este sentido, tan importante es reconocer las limitaciones cognitivas de los seres humanos a la hora de tomar decisiones como reconocer las limitaciones y potencialidades que establece la arquitectura de la elección en cada caso. Por ejemplo, la pretensión de reducir la emisión de gases de efecto invernadero y contribuir a no colapsar el tránsito en los desplazamientos dentro de la ciudad usando el transporte público en lugar de un vehículo particular debe contar con la existencia de una red de transporte público que sea realmente efectiva. Si esta red no existe, o existe en unas condiciones precarias, entonces la decisión a favor de un uso más racional y menos contaminante del espacio público queda fuertemente condicionada. Otro ejemplo sugerido por Gerald Dworkin -la prohibición de los duelos- ilustra la potencia de la arquitectura de la elección ${ }^{34}$. La institución de los duelos marcó durante siglos la manera en que un hombre debía demostrar su coraje si su honorabilidad era puesta en duda. Su ilegalización contribuyó a que la práctica se fuera extinguiendo poco a poco, si bien con dificultades, pero logrando en último término que muchos hombres no se vieran forzados a poner la vida en juego para demostrar su coraje e integridad.

33 Ibidem, p. 40.

${ }^{34}$ Dworkin, G. «Paternalism», en Sartorius, R. Paternalism, op. cit., p. 31. 
Las posibilidades de aplicación del paternalismo libertario y sus herramientas de economía conductual al ámbito del consumo son claras. Es posible mejorar las elecciones de los consumidores en muchos sentidos, mediante una buena divulgación, recordatorios pertinentes, estrategias de enfriamiento, incentivación de una compra reflexiva, etc. No obstante, es poco probable que sean las empresas comerciales en su conjunto las que estén potencialmente interesadas en ello ${ }^{35}$, entre otras razones porque un consumo reflexivo podría significar probablemente un consumo menor. En cambio, los poderes públicos pueden hacerlo y, normalmente, lo hacen promoviendo regulaciones del ámbito comercial para evitar prácticas engańosas o abusivas, su supervisión y sanción en los casos que sean necesarios, así como la creación de agencias de atención y protección del consumidor. Por poner un ejemplo concreto, la agencia catalana de consumo de la Generalitat de Catalunya difunde una definición de consumo responsable como aquella práctica adquisitiva que es racional, solidaria, sostenible, de proximidad y comprometida en la equidad de género. En su página web, se define la compra reflexiva como aquélla en la cual «el comprador prevé con anterioridad qué producto quiere adquirir y, en la mayoría de los casos, qué característica debe tener, como la marca, la cantidad o el precio». En este mismo contexto, se añaden unas advertencias de sentido común que constituyen un recordatorio en la línea de reforzar el Sistema 2: «Hay que controlar los gastos y evitar 'sobreendeudarse'. Planificar cómo se gasta y se ahorra el dinero permite saber adónde van a parar los ingresos y si se es capaz de reducir los gastos muy elevados o de disponer de un ahorro determinado para hacer frente a imprevistos ${ }^{36}$.

Por otra parte, las asociaciones de defensa de los consumidores y los medios de comunicación también pueden llevar a cabo una labor importante mediante la divulgación de recordatorios y advertencias con objeto de que la adquisición de bienes y servicios se haga en las condiciones adecuadas y no acabe siendo frustrante $o$ mostrando casos en que se han cometido fraudes explotando la ingenuidad de los consumidores. En este sentido, la mala publicidad puede generar correcciones importantes: «Un único y bien publicitado caso de un cliente insatisfecho, un producto de baja calidad peligroso o un desastre ambiental pueden hacer mucho por deshacer el duro trabajo de años para desarrollar un producto y construir una imagen ${ }^{37}$.

Desde luego, una difusión de este tipo de malas prácticas podría servir para que las empresas mejorasen sus estándares de atención, servicio y atención al cliente, si bien es cierto que, a menudo, como lamentan las organizaciones de defensa de los consumidores, éstos se contentan muy fácilmente con las disculpas y las bellas racionalizaciones publicitarias que se ofrecen desde los equipos de relaciones públicas de las empresas cuestionadas. La monitorización y la divulgación de las malas prácticas comerciales de las empresas y los gobiernos llevadas a cabo por las orga-

35 Cf. Gabriel, Y. y Lang, T. The Unmanageable Consumer, Sage, Londres, 2006, pp. 112133.

${ }^{36}$ Agència Catalana de Consum, http://www.consum.gencat.cat [consultado el 7 de septiembre de 2018].

${ }^{37}$ Gabriel, Y. y Lang, T. The Unmanageable Consumer, op. cit., p. 132. 
nizaciones de defensa de los consumidores y los medios de comunicación no pueden en ningún caso detenerse. Hay mucha gente interesada en que esa labor no sea eficaz, se empantane o no tenga la suficiente visibilidad. En definitiva, hay mucha gente que gana dinero intentando lograr que no prestemos demasiada atención cuando gastamos el nuestro.

\section{UN ARGUMENTO CONTRA EL PATERNALISMO LIBERTARIO}

Pese a sus inequívocas virtudes, el paternalismo libertario topa con tres fuentes de problemas potenciales. En primer lugar, está la cuestión de la transparencia de las medidas paternalistas; en segundo lugar, el riesgo de que los ciudadanos sobre los que se aplica una legislación inspirada en el paternalismo libertario sean manipulados; y, en tercer lugar, la cuestión de reconocer las exigencias legítimas del Sistema $1^{38}$. Con la pretensión de dejar para la parte final de este trabajo la discusión del argumento mencionado, que tiene que ver específicamente con el asunto de la transparencia, procederemos a analizar estas problemáticas en orden inverso al de su presentación.

Es posible creer que lo que hace que la vida valga la pena tenga que ver con atender a las demandas legítimas del Sistema 1 en lugar de recelar de ellas. Para expresarlo en términos coloquiales: a pesar de que puede ponernos en riesgo, el dicharachero Sistema 1 nos alegra el día, mientras que el antipático Sistema 2 nos aguarda por la noche para sermonearnos y censurarnos. En cualquier caso, los deseos y los impulsos irreflexivos que emergen del Sistema 1 alimentan la motivación de la gente. Y algunas veces, no siempre, desde luego, la motivación, sin ningún cálculo de probabilidades, conduce a las personas a hacer cosas extraordinariamente buenas. En eso consiste el coraje, por ejemplo. El escritor alemán Johann Wolfgang von Goethe definió el coraje como el compromiso de empezar algo sin disponer de ninguna garantía de éxito. No parece que el valor del coraje tenga nada que ver con el tipo de comportamiento que recomienda el Sistema 2 y no por ello se cuestiona el carácter de su excelencia. Con respecto a las demandas legítimas del Sistema 1, Sunstein reflexiona que el paternalismo libertario no tiene la intención de amargarle la vida a nadie, sino que, más bien, trata de hacer reflexionar a la gente acerca de los peligros de la falta de autocontrol, la presencia indetectable de los sesgos y la atención concentrada en el corto plazo. En este sentido, sostiene que

el problema efectivo yace no en alguna cuestión de grandes principios, sino en identificar qué quiere o quisiera la gente y en decidir si los arquitectos de la elección pueden ser fiables. Tal vez los arquitectos de la elección no saben qué desearía la gente si estuviera correctamente informada y quizás sus propias ideas no sean puras ${ }^{39}$.

${ }^{38}$ Cf. Sunstein, C. Paternalismo libertario. ¿Por qué un empujoncito?, op. cit., p. 169.

39 Ibidem, p. 183. 
Según Sunstein, la política del paternalismo libertario no puede impedir completamente la posibilidad de la manipulación o los abusos de poder, si bien es cierto que está un poco mejor pertrechada para evitarlos. Por un lado, parece claro que los empujoncitos y las estrategias a favor de reforzar la reflexión pueden incentivar la deliberación y una actitud activa de las personas en relación con las medidas que se les apliquen. Por otro lado, como el paternalismo libertario no impone gravosos costes materiales sobre las elecciones de las personas, su implementación se atiene a un esquema de estricta reversibilidad sencilla $a^{40}$. A diferencia de legislaciones más invasivas, las medidas del paternalismo suave o libertario son fácilmente reversibles. No obstante, el hecho de que sean reversibles no elimina la posibilidad de manipulación. El caso de la gestión del pago de impuestos podría ser un ejemplo. En muchos países europeos, se ha ido facilitando cada vez más la gestión del pago de impuestos mediante una presentación telemática e individualizada de la renta anual. El proceso se ha automatizado de tal modo que la administración ya envía un borrador de la declaración de renta que el contribuyente tiene la opción de confirmar o no. A pesar de que se ofrecen advertencias de que se revise el borrador exhaustivamente y se indiquen cambios en la situación fiscal antes de confirmarlo, la facilidad con la cual se puede solventar la tarea y la perspectiva de una devolución rápida logran que mucha gente opte por la vía menos engorrosa y le dé el visto bueno al borrador sin demorarse. Ahora bien, el borrador no siempre es correcto porque a veces no contiene todos los datos relevantes que solo conocen los contribuyentes y, a menudo, éstos ignoran la existencia de deducciones específicas -como las derivadas de pagar cuotas sindicales o de los gastos de movilidad generados por aceptar una ocupación lejana a la zona de residencia habitual- que les podrían reportar una devolución mayor. En este caso, la arquitectura de la elección diseñada por los poderes públicos incorpora las advertencias necesarias, facilita mucho la gestión global -evita desplazamientos, acumulaciones de papeles, aglomeraciones en las oficinas de gestión tributaria- y preserva la libertad de elección en última instancia, pero la misma sencillez y velocidad del proceso coadyuva a la inercia irreflexiva de los contribuyentes y, en consecuencia, no facilita suficientemente una elección consciente y soberana. Finalmente, está la cuestión de la transparencia de las medidas paternalistas. Sunstein defiende inequívocamente que las medidas paternalistas de un gobierno, sean éstas duras o suaves, ya se trate de mandatos y prohibiciones como de empujoncitos, deben ser transparentes.

El paternalismo suave, los empujoncitos y cualquier otro enfoque informado de modo conductual, no menos que el paternalismo duro, debe ser visible, escudrińado y monitoreado. En la medida de lo posible, las prácticas que encarnan el paternalismo suave deben estar sujetas, con anterioridad, al escrutinio público, a menudo a través de la normativa de aviso y comentario ${ }^{41}$.

\footnotetext{
40 Ibidem, pp. 177-179.

${ }^{41}$ Ibidem, p. 173.
} 
Pero la transparencia tal vez no sea una virtud, sino un defecto. Algunos críticos del enfoque político de la economía conductual mencionan que la transparencia de las medidas del paternalismo suave, precisamente debido a su suavidad, puede convertirlas en casi invisibles, no susceptibles de atención por parte de aquéllos sobre quienes se aplican, lo cual podría abrir la puerta a una manipulación de los ciudadanos por parte de los funcionarios del gobierno. Edward Glaeser, por ejemplo, sugiere que mientras el perfil nítido del paternalismo duro, con sus mandatos y prohibiciones, lo convierte en un objeto más fácil de controlar y supervisar, eso no resulta tan sencillo con el paternalismo suave debido a su carácter mucho más sutil ${ }^{42}$. En cualquier caso, la transparencia es exigible a los funcionarios porque forma parte de su responsabilidad pública. Lo que cabe tener presente en este punto es que el problema de la elección pública es, en realidad, un problema doble: por una parte, existe la posibilidad de que los políticos elegidos puedan verse influidos por grupos de presión privados cuyo objetivo es orientar la acción gubernamental en una línea que sea conforme a sus intereses; pero, por otra parte, no hay que olvidar que los legisladores y los políticos que diseñan e implementan las medidas paternalistas también son seres humanos cuyo razonamiento, como el de los ciudadanos comunes, está igualmente sometido a sesgos sistemáticos.

Pero, en relación con la elección pública, emerge otra dificultad adicional que Sunstein parece no haber tenido explícitamente en cuenta. El examen de esta dificultad permite generar un argumento contra el paternalismo libertario. La dificultad es la siguiente: un cuerpo de funcionarios electos puede promover medidas asociadas con el paternalismo suave con el objetivo último de legitimarse y perpetuarse en el poder. En este caso existe un incentivo racional para que ese cuerpo de funcionarios dedique esfuerzos de carácter paternalista al bienestar de los ciudadanos durante el periodo en que se mantiene en el cargo e incluso para intensificarlos a la vista de la celebración de unas próximas elecciones. Podemos imaginar que, en el caso abstracto así formulado, no se está produciendo ni la influencia de un grupo privado externo al gobierno con intención de condicionarlo a su favor ni tampoco el influjo de sesgos no conscientes en la toma de decisiones por parte de los funcionarios, si bien es cierto que, en el mundo social y político real, ambas cosas suceden todo el tiempo. Pero lo más relevante es que tampoco existe una motivación ilícita por parte de los funcionarios. Lo que el caso presenta es una actuación deliberada de la institución, ocupada por un partido o una coalición de partidos, que persigue su interés legítimo de continuar ejerciendo el poder y que, de manera subsidiaria, aunque no explícita, tiene en cuenta el bienestar de los ciudadanos.

Naturalmente, la racionalidad y la legitimidad asociadas a esta clase de actuaciones no pueden evitar que, a veces, se produzcan usos instrumentales o cínicos en los cuales el fin se convierte en medio: la promoción y la garantía del bienestar de los ciudadanos, que debería ser el fin de los funcionarios gubernamenta-

42 Glaeser, E. «Paternalism and Psychology», en University of Chicago Law Review, 73(1), 2006, pp. 133-156. 
les, acaba siendo en cambio un medio para su justificación y mantenimiento en el poder. Sunstein había argumentado que el escrutinio público de las medidas que un gobierno se dispone a aplicar, por muy paternalista libertario que sea, debería bastar para impedir el riesgo de las manipulaciones. No obstante, como es bien sabido, las administraciones estatales y regionales de las zonas ricas del mundo, como sucede en el caso de la Unión Europea, exhiben una gran capacidad de medios y recursos humanos para presentar sus propuestas de manera adecuada y persuasiva. En este sentido, se han destacado en las llamadas políticas del "globo sonda». A menudo, han lanzado propuestas legislativas al espacio público solo con el objeto de calibrar el efecto que generan a través de sus herramientas demoscópicas. Una vez evaluada la reacción de las poblaciones, tales administraciones pueden permitirse seguir adelante, ya sea corrigiendo, matizando o incluso descartando la propuesta inicial en favor de otra que sí parece que puede contar con el asentimiento del público y, por tanto, con la legitimación de su trabajo. En suma, si el objetivo real y verificable de un gobierno paternalista libertario no es en primer término el bienestar de los ciudadanos tal como éstos lo querrían, sino su propia conservación, entonces la propuesta de un gobierno paternalista que genera medidas bienintencionadas y respeta la libertad de elección queda cuestionada.

\section{CONCLUSIÓN}

Este trabajo ha realizado un breve examen de algunos de los hallazgos de la economía conductual para observar sus aplicaciones a los ámbitos del consumo y la política. Se ha verificado que la distinción entre un yo que experimenta y un yo que recuerda, junto con las aportaciones de la sociología y la antropología del consumo, puede ser empleada como una estructura básica para la explicación del comportamiento de los consumidores en sus prácticas de consumo cotidianas. Por otro lado, se ha comprobado que el refuerzo institucional al yo que recuerda constituye una estrategia básica de las políticas del paternalismo suave, las cuales han sido evaluadas como una contribución al debate no solo sobre el concepto de paternalismo, sino también sobre la justificación de un gobierno que se acredite como paternalista libertario. Por último, se ha presentado un argumento crítico contra el paternalismo libertario según el cual un gobierno podría aplicar medidas paternalistas libertarias con objeto de promover el bienestar de los ciudadanos, aunque no dejaría de ser racional su actuación si ocultara que, tras ellas, su finalidad prioritaria es la conservación del poder. 
